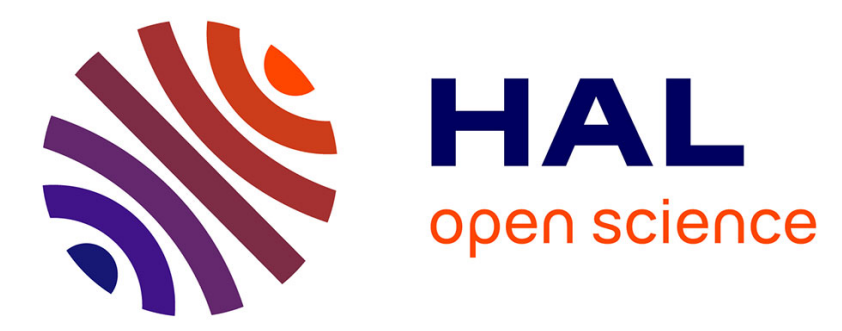

\title{
Bridging High-Level Synthesis and Application-Specific Arithmetic: The Case Study of Floating-Point Summations
}

\author{
Yohann Uguen, Florent de Dinechin, Steven Derrien
}

\section{To cite this version:}

Yohann Uguen, Florent de Dinechin, Steven Derrien. Bridging High-Level Synthesis and ApplicationSpecific Arithmetic: The Case Study of Floating-Point Summations. 27th International Conference on Field-Programmable Logic and Applications (FPL), IEEE, Sep 2017, Gent, Belgium. pp.8. hal$01373954 \mathrm{v} 2$

\section{HAL Id: hal-01373954 \\ https://hal.inria.fr/hal-01373954v2}

Submitted on 11 Sep 2017

HAL is a multi-disciplinary open access archive for the deposit and dissemination of scientific research documents, whether they are published or not. The documents may come from teaching and research institutions in France or abroad, or from public or private research centers.
L'archive ouverte pluridisciplinaire HAL, est destinée au dépôt et à la diffusion de documents scientifiques de niveau recherche, publiés ou non, émanant des établissements d'enseignement et de recherche français ou étrangers, des laboratoires publics ou privés. 


\section{Bridging High-Level Synthesis and Application-Specific Arithmetic: The Case Study of Floating-Point Summations}

\author{
Yohann Uguen \\ Univ Lyon, INSA Lyon, Inria, CITI \\ F-69621 Villeurbanne, France \\ Yohann.Uguen@insa-lyon.fr
}

\author{
Florent de Dinechin \\ Univ Lyon, INSA Lyon, Inria, CITI \\ F-69621 Villeurbanne, France \\ Florent.de-Dinechin@insa-lyon.fr
}

\author{
Steven Derrien \\ University Rennes 1, IRISA \\ Rennes, France \\ Steven.Derrien@univ-rennes1.fr
}

\begin{abstract}
FPGAs are well known for their ability to perform non-standard computations not supported by classical microprocessors. Many libraries of highly customizable applicationspecific IPs have exploited this capablity. However, using such IPs usually requires handcrafted HDL, hence significant design efforts. High Level Synthesis (HLS) lowers the design effort thanks to the use of $\mathrm{C} / \mathrm{C}++$ dialects for programming FPGAs. However, high-level $\mathrm{C}$ language becomes a hindrance when one wants to express non-standard computations: this languages was designed for programming microprocessors and carries with it many restrictions due to this paradigm. This is especially true when computing with floating-point, whose data-types and evaluation semantics are defined by the IEEE-754 and C11 standards. If the high-level specification was a computation on the reals, then HLS imposes a very restricted implementation space.
\end{abstract}

This work attempts to bridge FPGA application-specific efficiency and HLS ease of use. It specifically targets the ubiquitous floating-point summation-reduction pattern. A source-tosource compiler transforms selected floating-point additions into sequences of simpler operators using non-standard arithmetic formats. This improves performance and accuracy for several benchmarks, while keeping the ease of use of a high-level $\mathrm{C}$ description.

\section{INTRODUCTION}

Many case studies have demonstrated the potential of FieldProgrammable Gate Arrays (FPGAs) as accelerators for a wide range of applications, from scientific or financial computing to signal processing and cryptography. FPGAs offer massive parallelism and programmability at the bit level. These FPGAs characteristics enables programmers to exploit a range of techniques that avoid many bottlenecks of classical von Neumann computing: dataflow operation without the need of instruction decoding; massive register and memory bandwidth, without contention on a register file and single memory bus; operators and storage elements tailored to the application in nature, number and size.

However, to unleash this potential, development costs for FPGAs are orders of magnitude higher than classical programming. High performance and high design costs are the two faces of the same coin.

Hardware design flow and High-level synthesis: To address this, languages such as C or Java are increasingly being considered as hardware description languages. This has many advantages. The language itself is more widely known than any HDL. The sequential execution model makes designing and debugging much easier. One can even use software execution on a processor for simulation. All this drastically reduces development time.

The process of compiling a software program into hardware is called High-Level Synthesis (HLS), with tools such as Vivado HLS [11] or Catapult $\mathrm{C}^{1}$ among others [18]. These tools are in charge of turning a $\mathrm{C}$ description into a circuit. This task requires to extract parallelism from sequential programs constructs (e.g. loops) and expose this parallelism in the target design. Today's HLS tools are reasonably efficient at this task, and can automatically synthesize highly efficient pipelined dataflow architectures.

They however miss one important feature: they are not able to tailor operators to the application in size, and even less in nature. This comes from the $\mathrm{C}$ language itself: its high-level datatypes and operators are limited to a small number (more or less matching the hardware operators present in mainstream processors). Indeed, such high-level languages were designed to be compiled and run on hardware, not to describe hardware.

However, HLS tool know a lot about the context of each operator. This should allow them to transform these simple operators into application-specific ones, thus exploiting FPGAs to their full potential. The broader objective of this work is to demonstrate this opportunity. For this purpose, we envision a compilation flow involving one or several source-to-source transformations, as illustrated by Figure 1 .

Arithmetic in HLS: To better exploit the freedom offered by hardware and FPGAs, HLS vendors have enriched the C language with integer and fixed-point types of arbitrary size ${ }^{2}$. However the operations on these types remain limited to the basic arithmetic and logic ones. Exotic or complex operators (for instance for finite fields) may be encapsulated in a $\mathrm{C}$ function that is called to instantiate the operator.

\footnotetext{
${ }^{1}$ Catapult C Synthesis, Mentor Graphics, 2011, http://calypto.com/en/ products/catapult/overview/

${ }^{2}$ Arbitrary-size floating-point should follow some day, it is well supported by mature libraries and tools
} 


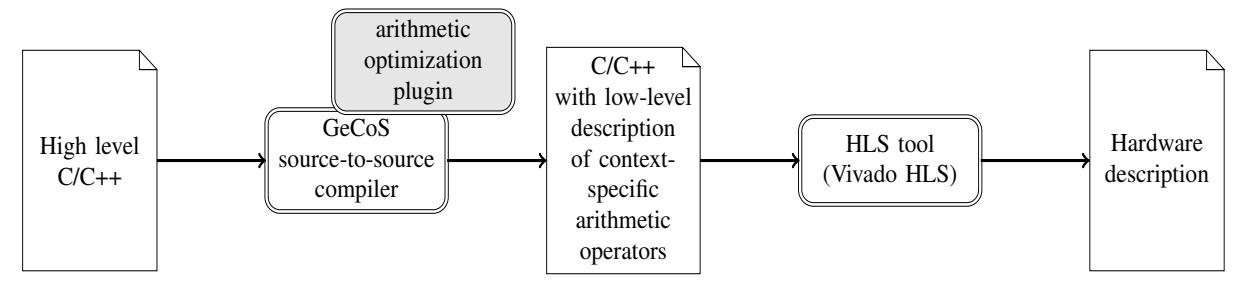

Figure 1: The proposed compilation flow

The case study in this work is a program transformation that applies to floating-point additions on a loop's critical path. It decomposes them into elementary steps, resizes the corresponding sub-components to guarantee some userspecified accuracy, and merges and reorders these components to improve performance. The result of this complex sequence of optimizations could not be obtained from an operator generator, since it involves global loop information.

Before detailing it, we must digress a little on the subtleties of the management of floating-point arithmetic by compilers.

HLS faithful to the floats: Most recent compilers, including the HLS ones [10], attempt to follow established standards, in particular C11 and, for floating-point arithmetic, IEEE-754. This brings the huge advantage of almost bit-exact reproducibility - the hardware will compute exactly the same results as the software. However, it also greatly reduces the freedom of optimization by the compiler. For instance, as floating point addition is not associative, C11 mandates that code written $a+b+c+d$ should be executed as $((a+b)+c)+d$, although $(a+b)+(c+d)$ would have shorter latency. This also prevents the parallelization of loops implementing reductions. A reduction is an associative computation which reduces a set of input values into a reduction location. Listing 1 provides the simplest example of reduction, where acc is the reduction location.

The first column of Table I shows how Vivado HLS synthesizes Listing 1 on Kintex7. The floating-point addition takes 7 cycles, and the adder is only active one cycle out of 7 due to the loop-carried dependency. Listing 2 shows a different version of Listing 1 that we coded such that Vivado HLS expresses more parallelism. Vivado HLS will not transform Listing 1 into Listing 2, because they are not semantically equivalent ${ }^{3}$ (the floating-point additions are reordered as if they were associative). However, the tool is able to exploit the parallelism in Listing 2 (second column of Table I): The main adder is now active at each cycle on a different sub-sum.

Note that Listing 2 is only here as an example and might need more logic if $\mathrm{N}$ was not a multiple of 10 .

Towards HLS faithful to the reals: Another point of view, chosen in this work, is to assume that the floatingpoint $\mathrm{C}$ program is intended to describe a computation on real numbers when the user specifies it. In other words, the floats are interpreted as real numbers in the initial C,

\footnotetext{
${ }^{3} \mathrm{~A}$ parallel execution with the sequential semantics is also possible, but very expensive [13].
}

Listing 1: Naive reduction

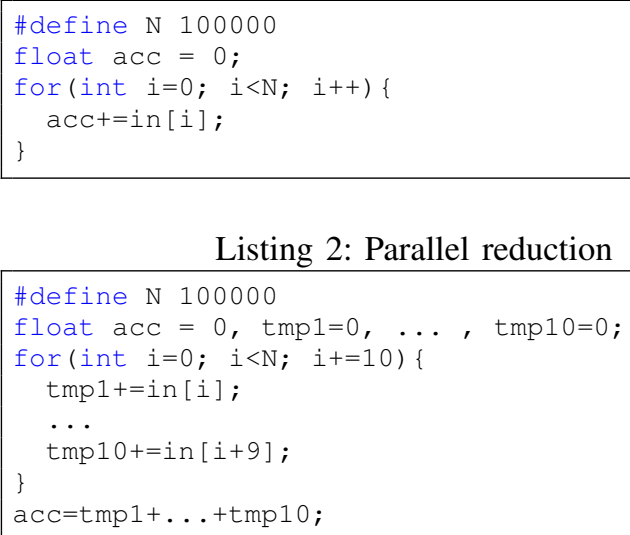

Listing 2: Parallel reduction

thus recovering the freedom of associativity (among other). Indeed, most programmers will perform the kind of nonbit-exact optimizations illustrated by Listing 2 (sometimes assisted by source-to-source compilers or "unsafe" compiler optimizations). In a hardware context, we may also assume they wish they could tailor the precision (hence the cost) to the accuracy requirements of the application - a classical concern in HLS [9], [2]. In this case, a pragma should specify the accuracy of the computation with respect to the exact result. A high-level compiler is then in charge of determining the best way to ensure the prescribed accuracy.

The proposed approach uses number formats that are larger or smaller than the standard ones. These, and the corresponding operators, are presented in Section II. The contribution of this paper, which are compiler transformations to generate $\mathrm{C}$ description of these operators in a HLS workflow, is presented in Section III. Section IV evaluates our approach on the FPMark benchmark suite.

\section{THE ARITHMETIC SIDE: AN APPLICATION-SPECIFIC ACCUMULATOR IN VIVADO HLS}

The accumulator that we used for this paper is based on a more general idea developed by Kulisch. He advocated a very large floating-point accumulator [14] whose 4288 bits would cover the entire range of double precision floating-point. Such an accumulator would remove rounding errors from all the possible floating-point additions and sums of products, with the added bonus that addition would become associative. 
So far, Kulisch's full accumulator has proven too costly to appear in mainstream processors. However, in the context of application acceleration with FPGAs, it can be tailored to the accuracy requirements of applications. Its cost then becomes comparable to classical floating point operators, although it vastly improves accuracy [6]. This operator can be found in the FloPoCo [5] generator and in Altera DSP Builder Advanced. Its core idea, illustrated on Figure 2, is to use a large fixed-point register into which the mantissas of incoming floating-point summands are shifted (top) then accumulated (middle). A third component (bottom) converts the content of the accumulator back to the floating-point format. The sub-blocks visible on this figure (shifter, adder, and leading zero counter) are essentially the building blocks of a classical floating-point adder.
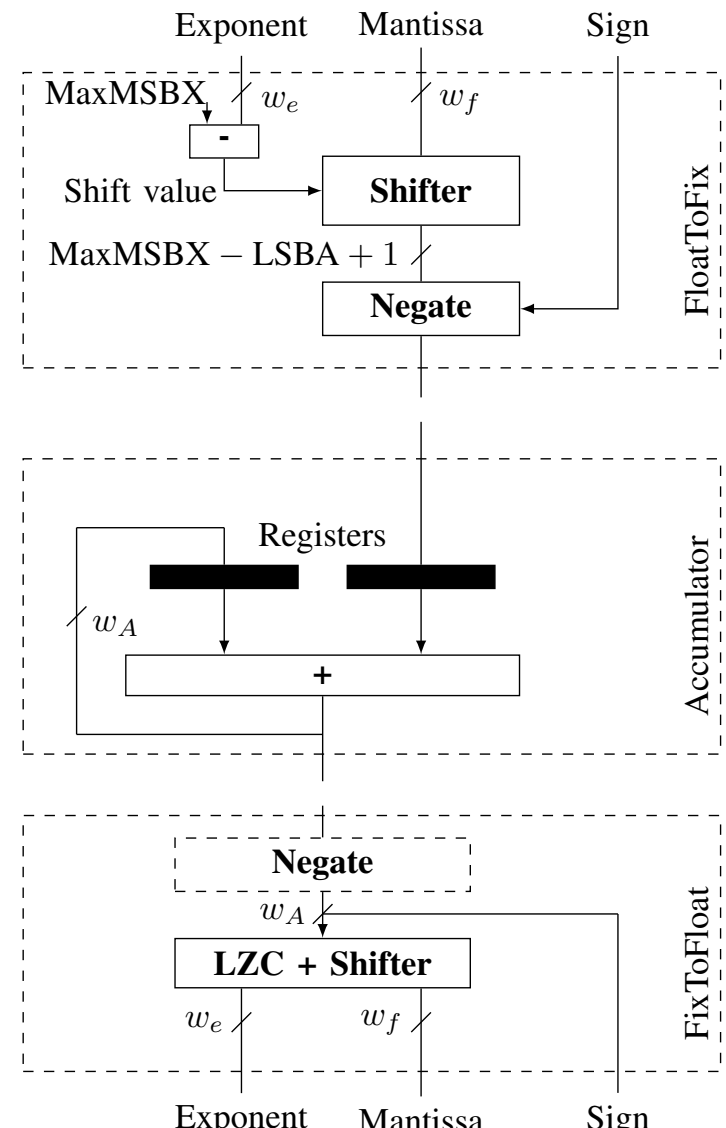

Figure 2: The conversion from float to fixed-point (top), the fixed-point accumulation (middle) and the conversion from the fixed-point format to a float (bottom).

The accumulator used here slightly improves the one offered by FloPoCo [6]:

- It supports subnormal numbers [17].

- In FloPoCo, FloatToFix and Accumulator form a single component, which restricts its application to simple accumulations similar to Listing 1 . The two components of Figure 2 enable a generalization to arbitrary summations within a loop, as Section III will show.

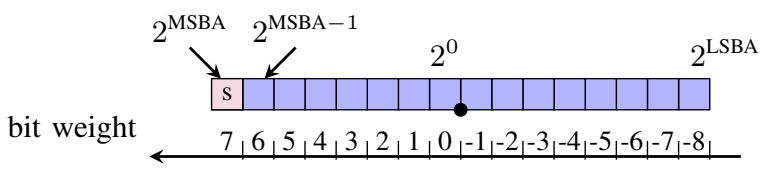

Figure 3: The bits of a fixed-point format, here $(\mathrm{MSBA}, \mathrm{LSBA})=(7,-8)$.

Note that we could have implemented any other nonstandard operator performing a reduction such as [16], [12].

\section{A. The parameters of a large accumulator}

The main feature of this approach is that the internal fixed-point representation is configurable in order to control accuracy. It has two parameters:

- MSBA is the weight of the most significant bit of the accumulator. For example, if MSBA $=20$, the accumulator can accommodate values up to a magnitude of $2^{20} \approx 10^{6}$.

- LSBA is the weight of the least significant bit of the accumulator. For example, if LSBA $=-50$, the accumulator can hold data accurate to $2^{-50} \approx 10^{-15}$.

Such a fixed-point format is illustrated in Figure 3.

The accumulator width $w_{a}$ is then computed as MSBA LSBA +1 , for instance 71 bits in the previous example. 71 bits represents a wide range and high accuracy, and still additions on this format will have one-cycle latency for practical frequencies on recent FPGAs. If this is not enough the frequency can be improved thanks to partial carry save [6] but this was not useful in the present work. For comparison, for the same frequency, a floating-point adder has a latency of 7 to 10 cycles, depending on the target.

In the following, the latency of a circuit denotes the number of cycles needed for the entire application to complete.

\section{B. Implementation within a HLS tool}

This accumulator has been implemented in $\mathrm{C}$, using arbitrary-precision fixed point types (ap_int). The leading zero count, bit range selections and other operations are implemented using Vivado HLS built-in functions. For modularity purposes, the FloatToFix and FixToFloat are wrapped into $\mathrm{C}$ functions (respectively 28 and 22 lines of code). Their calls are inlined to enable HLS optimizations.

Because the internal accumulation is performed on a fixedpoint integer representation, the combinational delay between two accumulations is lower compared to a full floating point addition. HLS tools can take advantage of this delay reduction by more agressive loop pipelining (with shorter Initiation Interval), resulting in a design with a shorter overall latency.

\section{Validation}

To evaluate and refine this implementation, we used Listing 3 , which we compared to Listings 1 and 2 . In the latter, the loop was unrolled by a factor 7 , as it is the latency of a floating-point adder on our target FPGA (Kintex-7). 


\begin{tabular}{|c||c|c|c|c||c||c|}
\hline & $\begin{array}{c}\text { Listing 1 } \\
\text { (float) }\end{array}$ & $\begin{array}{c}\text { Listing 2 } \\
\text { (float) }\end{array}$ & $\begin{array}{c}\text { Listing 1 } \\
\text { (double) }\end{array}$ & $\begin{array}{c}\text { Listing 2 } \\
\text { (double) }\end{array}$ & $\begin{array}{c}\text { Listing 3 } \\
\text { (71 bits) }\end{array}$ & $\begin{array}{c}\text { FloPoCo VHDL } \\
\text { (71 bits) }\end{array}$ \\
\hline LUTs & 266 & 907 & 801 & 2193 & 736 & 719 \\
\hline DSPs & 2 & 4 & 3 & 6 & 0 & 0 \\
\hline Latency & $700 \mathrm{~K}$ & $142 \mathrm{~K}$ & $700 \mathrm{~K}$ & $142 \mathrm{~K}$ & $100 \mathrm{~K}$ & $100 \mathrm{~K}$ \\
\hline Accuracy & 17 bits & 17 bits & 24 bits & 24 bits & 24 bits & 24 bits \\
\hline
\end{tabular}

Table I: Synthesis results of different accumulators using Vivado HLS for Kintex 7.

For test data, we use as in Muller et al. [17] the input values $c[i]=(f l o a t) \cos$ (i), where $i$ is the input array's index. Therefore the accumulation computes $\sum_{i} \mathrm{c}[\mathrm{i}]$.

The parameters chosen for the accumulator are:

- $\mathrm{MSBA}=17$. Indeed, as we are adding $\cos (i) 100 \mathrm{~K}$ times, an upper bound is $100 \mathrm{~K}$, which can be encoded in 17 bits.

- MaxMSBX $=1$ as the maximum input value is 1 .

- $\mathrm{LSBA}=-50$ : the accumulator itself will be accurate to the 50th fractional bit. Note that a float input will see its mantissa rounded by FloatToFix only if its exponent is smaller than $2^{-25}$, which is very rare. In other words, this accumulator is much more accurate than the data that is thrown to it.

The results are reported in Table I for simple and double precision. The Accuracy line of the table reports the number of correct bits of each implementation, after the result has been rounded to a float. All the data in this table was obtained by generating VHDL from C synthesis using Vivado HLS followed by place and route from Vivado v2015.4, build 1412921. This table also reports synthesis results for the corresponding FloPoCo-generated VHDL, which doesn't include the array management.

Vivado HLS uses DSPs to implement the shifts in its floating-point adders. Even if the shifts were implemented in LUTs, the first column would remain well below 500 LUTs: it has the best resource usage. However the latency of one iteration is 7 cycles, hence $100 \mathrm{~K}$ iterations takes $700 \mathrm{~K}$ cycles. When unrolling the loop, Vivado HLS is using almost 4 times more LUTs for floats, and 3 times more for doubles. The unrolled versions improves latency over naive versions. Nevertheless, the proposed approach gets even betters latencies for a reasonable LUT usage. It also achieves maximum accuracy for the float format, which caps at 24 bits (the internal representations of the double, unrolled double and proposed approach have a higher accuracy than 24 bits, but their result is then rounded to a float). Finally, our results are very close to FloPoCo ones, both in terms of LUTs usage, DPSs and latency.

Listing 3: Sum of floats using the large fixed-point accumulator

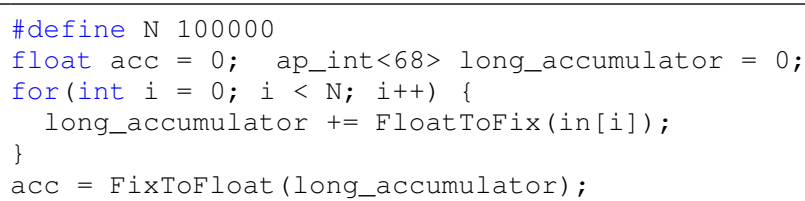

Using this implementation method, we also created an exact floating-point multiplier with the final rounding removed as in [6]. This function is called ExactProduct and represents 44 lines of code. Due to lack of space we do not present it in detail. As the output of this multiplier is not standard, we also created an adapted Float-to-fix block called ExactProductFloatToFix (21 lines of code).

\section{THE COMPILER SIDE: GeCOS SOURCE-TO-SOURCE TRANSFORMATIONS}

The previous section has shown that Vivado HLS can be used to synthesize very efficient specialized floating point operators which rival in quality with those generated by FloPoCo. Our goal is now to study how such optimization can be automated. More precisely, we aim at automatically optimizing Listing 1 into Listing 3, and generalizing this transformation to many more situations.

For convenience, this optimization was developed as a source-to-source transformation implemented within the open source $\mathrm{GeCoS}$ compiler framework [8]. It is publicly available with $\mathrm{GeCoS}$. Source-to-source compiler are very convenient in an HLS context, since they can be used as optimization frontends on top of closed-source commercial tools.

This work focuses on two computational patterns, namely the accumulation and the sum of product. Both are specific instances of the reduction pattern, which can be optimized by many compilers or parallel run-time environments. Reduction pattern are exposed to the compiler/runtime either though user directives (e.g \#pragma reduce in openMP), or automatically inferred using static analysis techniques [19], [7].

As the problem of detecting reductions is not the main focus on this work, our tool uses a straightforward solution to the problem using a combination of user directive and (simple) program analysis. More specifically, the user must identify a target accumulation variable through a pragma, and provide additional information such as the dynamic range of the accumulated data along with the target accuracy. In the future, we expect to improve the program analysis, so that the two later parameter could be omitted in some situations.

We found this approach easier, more general and less invasive than those attempting to convert a whole floatingpoint program into a fixed-point implementation [20].

\section{A. Proposed compiler directive}

In imperative languages such as $\mathrm{C}$, reductions are implemented using for or while loop constructs. Our compiler directive must therefore appear right outside such a construct. Listing 4 illustrates its usage on the code of Listing 1. 
The pragma must contain the following information:

- The keyword FPacC, which triggers the transformations.

- The name of the variable in which the accumulation is performed, preceded with the keyword VAR. In the example, the accumulation variable is acc.

- The maximum value that can be reached by the accumulator through the use of the MaxAcc keyword. This value is used to determine the weight MSBA.

- The desired accuracy of the accumulator using the epsilon keyword. This value is used to determine the weight LSBA.

- Optional: The maximum value among all inputs of the accumulator in the MaxInput field. This value is used to determine the weight MaxMSBX. If this information is not provided, then MaxMSBX is set to MSBA.

Listing 4: Illustration of the use of a pragma for the naive accumulation

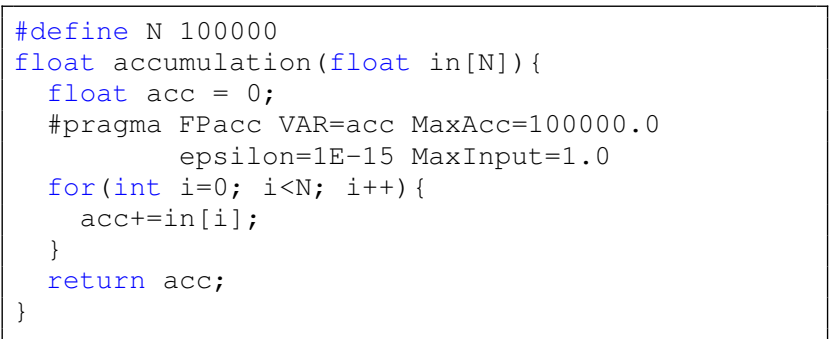

In the case when no size parameters are given, a full Kulisch accumulator is currently produced. Note that the user can quietly overestimate the maximum value of the accumulator without major impact on area. For instance, overestimating MaxAcC by a factor 10 only adds 3 bits to the accumulator width.

\section{B. Proposed code transformation}

The proposed transformation operates on the compiler program intermediate representation (IR), and rely on the ability to identify loops constructs and expose def/use relations between instructions of a same basic block in the form of an operation dataflow graph (DFG).

Listing 5: Simple reduction with multiple accumulation statements

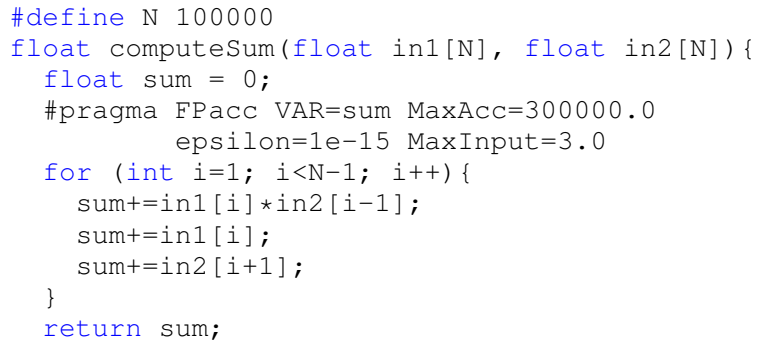

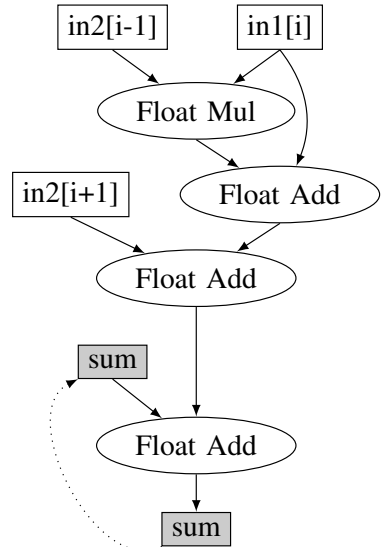

(a) Loop body dataflow graph

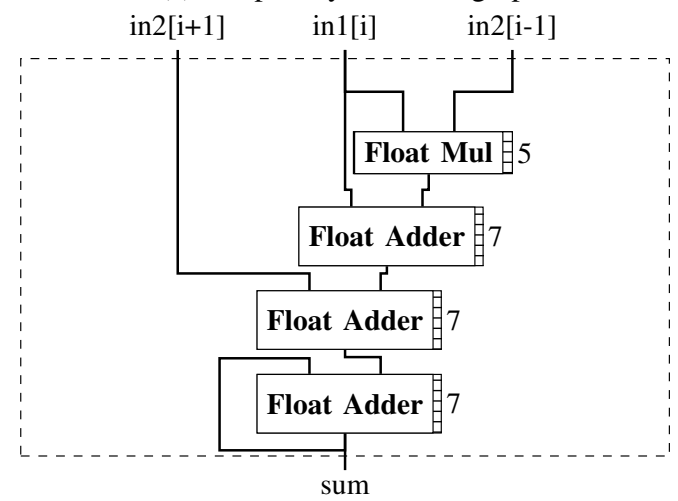

(b) Architecture

Figure 4: DFG of the loop body from Listing 5 (top) and its corresponding architecture (bottom). Keywords float mul and float add correspond to floating-point multipliers and adders respectively

To illustrate the transformation, consider the toy but nontrivial program of Listing 5. This program performs a reduction into the variable sum, involving both sums and sums of product operations. Figure $4 \mathrm{a}$ shows the operation dataflow graph for the loop body of this program. In this Figure, dotted arrows represent loop-carried dependencies between operations belonging to distinct loop iterations. Such loopcarried dependencies have a very negative impact on the kernel latency as they prevent loop pipelining. For example, when using a pipelined floating-point adder with a seven cycle latency, the HLS tool will schedule a new iteration of the loop at best every seven cycles.

As illustrated in Figure 5a, the proposed transformation hoists the floating-point normalization step out of the loop, and performs the accumulation using fixed point arithmetic. Since integer add operations can generally be implemented with a 1-cycle delay, the HLS tool may now be able to initiate a new iteration every cycle, improving the overall latency by a factor of 7 .

The code transformation first identifies all relevant basic blocks (i.e those associated to the pragma directive). It then 


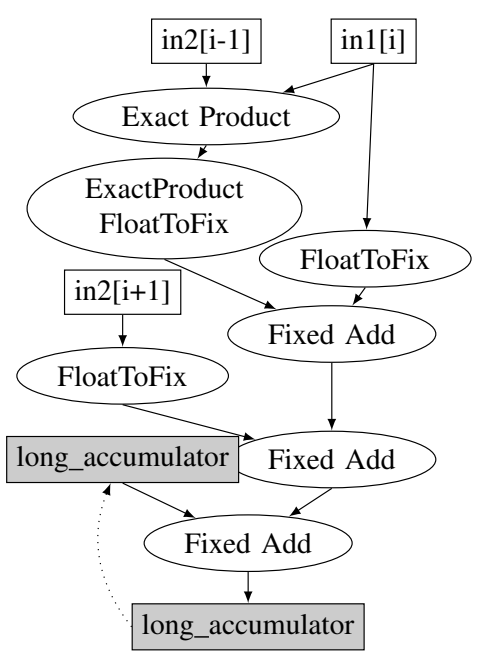

(a) Loop body dataflow graph

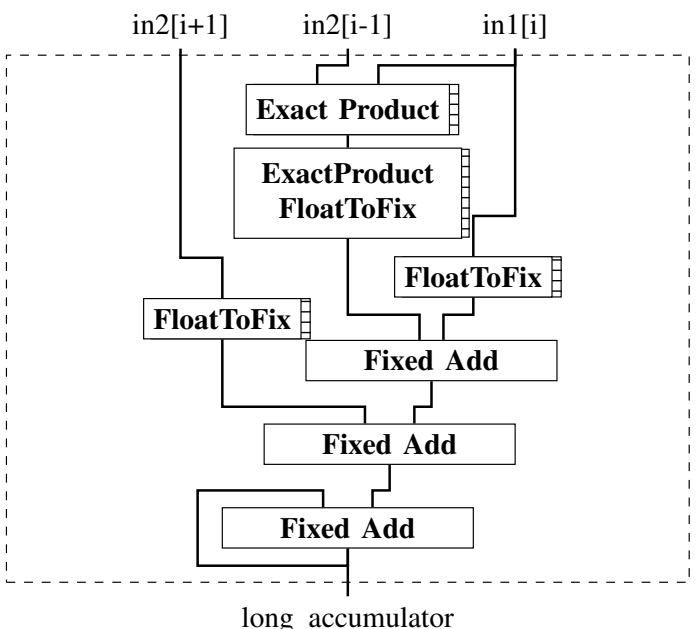

(b) Architecture

Figure 5: DFG of the loop body from Listing 5 (top) and its corresponding architecture (bottom) after transformations

performs a backward traversal of the dataflow graph, starting from a Float Add node that writes to the accumulation variable identified by the \#pragma.

During this traversal, the following actions are performed depending on the visited nodes:

- A node with the summation variable is ignored.

- A Float Add node is transformed to an accurate fixedpoint adder. The analysis is then recursively launched on that node.

- A Float Mul node is replaced with a call to the ExactProduct function followed by a call to ExactProdFloatToFix.

- Any other node has a call to FloatToFix inserted.

This algorithm rewrites the DFG from Figure 4a into the new DFG shown on Figure 5a. In addition, a new basic block containing a call to FixToFloat is inserted immediately after the transformed loop, in order to expose the floating point representation of the results to the remainder of the program.

\begin{tabular}{|c|c|c|c|c|}
\hline & Naive & $\begin{array}{c}\text { Transformed } \\
\text { LSBA }=-14\end{array}$ & $\begin{array}{c}\text { Transformed } \\
\text { LSBA }=-20\end{array}$ & $\begin{array}{c}\text { Transformed } \\
\text { LSBA }=-50\end{array}$ \\
\hline \hline LUTs & 538 & 693 & 824 & 1400 \\
\hline DSPs & 5 & 2 & 2 & 2 \\
\hline Latency & $2000 \mathrm{~K}$ & $100 \mathrm{~K}$ & $100 \mathrm{~K}$ & $100 \mathrm{~K}$ \\
\hline
\end{tabular}

Table II: Comparison between the naive code from Listing 5 and its transformed equivalent. All these versions run at $100 \mathrm{MHz}$.

From there, it is then possible to regenerate the corresponding $C$ code. As an illustration of the whole process, Figures $4 \mathrm{~b}$ and $5 \mathrm{~b}$ describe the architectures corresponding to the code before and and after the transformation.

\section{Evaluation of the toy example of Listing 5}

The proposed transformations work on non-trivial examples such as the one represented in Listing 5. Table II shows how resource consumption depends on epsilon, all the other parameters being those given in the pragma of Listing 5 . All these versions where synthesised for $100 \mathrm{MHz}$.

Compared to the classical IEEE-754 implementation, the transformed code uses more LUTs for less DSPs. This is due to Vivado implementing shifters using DSPs within the floatingpoint IP, but not in the transformed code. In all cases, on this example, the transformed code has its latency reduced by a factor 20 .

\section{EVALUATION ON FPMARK BENCHMARKS}

In order to evaluate the relevance of the proposed transformations on real-life programs, we used the EEMBC FPMark benchmark suite [1].

This suite consists of 10 programs. A first result is that half of these programs contain visible accumulations:

- Enhanced Livermore Loops (1/16 kernels contains one accumulation)

- LU Decomposition (multiple accumulations)

- Neural Net (multiple accumulations)

- Fourier Coefficients (one accumulation)

- Black Scholes (one accumulation)

The following focuses on these, and ignores the other half (Fast Fourier Transform, Horner's method, Linpack, ArcTan, Ray Tracer).

Most benchmarks come in single-precision and doubleprecision versions. We focus here on the single-precision. Double-precision benchmarks lead to the same conclusions.

\section{A. Benchmarks and accuracy: methodology}

Each benchmark comes with a golden reference against which the computed results are compared. As the proposed transformations are controlled by the accuracy, it may happen that the transformed benchmark is less accurate than the original. In this case, it will not pass the benchmark verification test, and rightly so.

A problem is that the transformed code will also fail the test if it is more accurate than the original. Indeed, the golden reference is the result of a certain combination of rounding 
errors using the standard FP formats, which we do not attempt to replicate.

To work around this problem, each benchmark was first transformed into a high-precision version where the accumulation variable is a 10,000-bit floating-point numbers using the MPFR library. We used the result of this highly-accurate version as a "platinum" reference, against which we could measure the accuracy of the benchmark's golden reference. This allowed us to choose our epsilon parameter such that the transformed code would be at least as accurate as the golden reference. This way, the epsilon of the following results is obtained through profiling. The accuracy of the obtained results are computed as the number of correct bits of the result.

We first present the benchmarks that are improved by our approach before discussing the reasons why we can't prove that the others are.

\section{B. Benchmarks improved by the proposed transformation}

Enhanced Livermore Loops: This program contains 16 kernels of loops that compute numerical equations. Among these kernels, there is one that performs a sum-of-product (banded linear equations). This kernel computes 20000 sumsof-products. The values accumulated are pre-computed. This is a perfect candidate for the proposed transformations.

For this benchmark, the optimal accumulation parameters were found as:

$\operatorname{MaxACC}=50000.0$ epsilon $=1 e-5$

MaxInput $=22000.0$

Synthesis results of both codes (before and after transformation) are given in Table III. As in the previous toy examples, latency and accuracy are vastly improved for comparable area.

\begin{tabular}{|c|c|c|c|c|c|}
\hline Benchmark & Type & LUTs & DSPs & Latency & Accuracy \\
\hline Livermore & Original & 384 & 5 & $80 \mathrm{~K}$ & 11 bits \\
& Transformed & 576 & 2 & $20 \mathrm{~K}$ & 13 bits \\
\hline \multirow{2}{*}{ LU-8 } & Original & 809 & 5 & 82 & $8-23$ bits \\
& Transformed & 1007 & 2 & 17 & 23 bits \\
\hline LU-45 & Original & 819 & 5 & 452 & $8-23$ bits \\
& Transformed & 1034 & 2 & 54 & 23 bits \\
\hline Scholes & Original & 15640 & 175 & N/A & 19 bits \\
& Transformed & 15923 & 175 & N/A & 23 bits \\
\hline Fourier & Original & 34596 & 64 & N/A & 6 bits \\
& Transformed & 34681 & 59 & N/A & 11 bits \\
\hline
\end{tabular}

Table III: Synthesis results of benchmarks before and after transformations

LU Decomposition and Neural Net: Both the LU decomposition and the neural net programs contain multiple nested small accumulations. In the LU decomposition program, an inner loop accumulates between 8 and 45 values. Such accumulations are performed more than $7 \mathrm{M}$ times. In the neural net program, inner loops accumulate between 8 and 35 values, and such accumulations are performed more than $5 \mathrm{~K}$ times.

Both of these programs accumulate values from registers or memory that are already computed. It makes these programs good candidates for the proposed transformations.
Vivado HLS is unable to predict a latency for these designs due to their non-constant loop trip counts. As a consequence, instead of presenting results for the complete benchmark, we restrict ourselves to the LU innermost loops. Table III shows the results obtained for the smallest ( 8 terms) and the largest (45 terms) sums-of-products in lines LU-8 and LU45 respectively. The latency is vastly improved even for the smallest one. The accuracy results of the original code here varies from 8 to 23 bits between different instances of the loops. To have a fair comparison, we generated a conservative design that performs 23 bits accuracy on all loops, using a sub-optimal amount of resources.

\section{Benchmarks that exposed the limitations of HLS tools}

Black Scholes: This program contains an accumulation that sums 200 terms. The result of this computation is divided by a constant (that could be optimized by using transformations based on [3]). This process is performed 5000 times.

Here the optimal accumulator parameters are the following: MaxAcC $=245000.0$ epsilon=1e-4

MaxInput $=278.0$

This gives us an accumulator that uses 19 bits for the integer part and 10 bits for the fractional part. The result of the synthesis are provided in Table III.

For comparable area, accuracy is vastly improved but latency could not be obtained from Vivado HLS. Indeed, the Black Scholes algorithm uses the mathematical function power. Such a function is not natively supported by Vivado HLS, and was therefore implemented by hand using a data dependant trip count loop. Because of this, the tool cannot statically derive the execution latency of the benchmark.

Fourier Coefficients: The Fourier coefficients program, which computes the coefficients of a Fourier series, contains an accumulation which is performed in single precision. This program comes in three different configurations: small, medium and big. Each of them computes the same algorithm but with a different amount of iterations. The big version is supposed to compute the most accurate answer. We obtain similar results for the three versions of this program, as a consequence we only present the big version here. In this version, there are multiple instances of $2 \mathrm{~K}$ terms accumulations. The accumulator is reset at every call.

The parameters determined for this benchmark were the following:

MaxAcC $=6000.0$ epsilon=1e-7 MaxInput $=10.0$

This results in an accumulator using 14 bits for the integer part and 24 bits for the fractional part. The synthesis results obtained for the original and transformed codes are given in Table III.

Here again, area cost is comparable, while accuracy is improved by 5 bits (which represents one order of magnitude). As for Black Scholes, Vivado HLS cannot compute the overall latency due to the power function. However, since our operators have a shorter latency by design, we expect the circuits to also have a shorter latency. 
The power operator could be implemented such as in [4] in the near future. This would allow us to obtain the latency of the two last benchmarks.

\section{CONCLUSION}

The main outcome of this work is an evidence that HLS tools have the potential to generate efficient designs for handling floating-point computations in a completely nonstandard way. Our results show that the use of applicationspecific intermediate formats can provide both performance and accuracy at a competitive cost. To achieve this, we have to sacrifice the strict respect of the IEEE-754 and C11 standards. It is replaced by the strict respect of a high-level accuracy specification.

Classically, designers face a trade-off between performance and cost. Our approach brings computation accuracy to this trade-off. Some designers may not like this. To convince them, consider that established performance benchmarks compute results which are accurate only to a few bits. If only a few bits are important, do we really need to instantiate 32-bit or 64-bit floating-point operators to compute them ? Isn't this accuracy information worth investigating and exploiting?

This work also provides a practical tool that improves a given $C$ program. The input to the tool is application-specific information representing high-level domain knowledge such as the range and desired accuracy of a variable. The resulting code is compatible with Vivado HLS.

The proposed transformation already works very well on all the FPMarks that contains a reduction where it improves both latency and accuracy by an order of magnitude for comparable area.

In the longer term, we believe there is much more to come. The arithmetic optimizations that a classical compiler can do are very limited by the fixed hardware of classical processors. With compilers of high-level software to hardware, there is much more freedom, hence many more opportunities to build application-specific arithmetic operators.

Future work will attempt to explore this new realm, starting with operator specialization; operator fusion such as in [21] but at a more coarse grain allowing more aggressive fusion; compile-time generation of application-specific cores; error analysis such as in [15] benefiting from compilers static analysis and more generaly building upon compiler progresses in program analysis.

\section{REFERENCES}

[1] EEMBC - the embedded microprocessor benchmark consortium. http: //www.eembc.org/.

[2] G. Caffarena, J. A. Lopez, C. Carreras, and O. Nieto-Taladriz. High-level synthesis of multiple word-length DSP algorithms using heterogeneousresource FPGAs. In Field Programmable Logic and Applications, pages $1-4,2006$.

[3] F. de Dinechin and L-S. Didier. Table-Based Division by Small Integer Constants, pages 53-63. 2012.

[4] F. de Dinechin, P. Echeverria, M. Lopez-Vallejo, and B. Pasca. FloatingPoint Exponentiation Units for Reconfigurable Computing. ACM Transactions on Reconfigurable Technology and Systems, pages 4:1-4:15, 2013.

[5] F. de Dinechin and B. Pasca. High-Performance Computing Using FPGAs, chapter Reconfigurable Arithmetic for High-Performance Computing, pages 631-663. 2013.

[6] F. de Dinechin, B. Pasca, O. Creţ, and R Tudoran. An FPGA-specific approach to floating-point accumulation and sum-of-products. In FieldProgrammable Technologies, pages 33-40. IEEE, 2008.

[7] J. Doerfert, K. Streit, S. Hack, and Z. Benaissa. Polly's polyhedral scheduling in the presence of reductions. International Workshop on Polyhedral Compilation Techniques, 2015.

[8] A. Floc'h, T. Yuki, A El-Moussawi, A. Morvan, K Martin, M. Naullet, M. Alle, L. L'Hours, N. Simon, S. Derrien, F. Charot, C. Wolinski, and O Sentieys. GeCoS: A framework for prototyping custom hardware design flows. In Source Code Analysis and Manipulation, pages 100$105,2013$.

[9] M. Gort and J. H. Anderson. Range and bitmask analysis for hardware optimization in high-level synthesis. In Asia and South Pacific Design Automation Conference, pages 773-779, 2013.

[10] J. Hrica. Floating-point design with vivado HLS, 2012. Xilinx Application Note.

[11] Xilinx Inc. Vivado Design Suite User Guide: High-Level Synthesis. 2015.

[12] E. Kadric, P. Gurniak, and A. DeHon. Accurate parallel floating-point accumulation. IEEE Transactions on Computers, pages 3224-3238, 2016.

[13] N. Kapre and A. DeHon. Optimistic parallelization of floating-point accumulation. In Symposium on Computer Arithmetic, pages 205-216, 2007.

[14] U. Kulisch and V. Snyder. The exact dot product as basic tool for long interval arithmetic. Computing, pages 307-313, 2011.

[15] M. Langhammer and T. VanCourt. FPGA floating point datapath compiler. In Field Programmable Custom Computing Machines, pages 259-262, 2009.

[16] Z. Luo and M. Martonosi. Accelerating pipelined integer and floatingpoint accumulations in configurable hardware with delayed addition techniques. IEEE Transactions on Computers, pages 208-218, 2000.

[17] J-M. Muller, N. Brisebarre, F. de Dinechin, C-P. Jeannerod, V. Lefèvre, G. Melquiond, N. Revol, D. Stehlé, and S. Torres. Handbook of Floating-Point Arithmetic. Birkhäuser Boston, 2010.

[18] R. Nane, V. M. Sima, C. Pilato, J. Choi, B. Fort, A. Canis, Y. T. Chen, H. Hsiao, S. Brown, F. Ferrandi, J. Anderson, and K. Bertels. A survey and evaluation of fpga high-level synthesis tools. Computer-Aided Design of Integrated Circuits and Systems, pages 1591-1604, 2016.

[19] X. Redon and P. Feautrier. Detection of scans in the polytope model. Parallel Algorithms and Applictations, pages 229-263, 2000.

[20] O. Sentieys, D. Menard, D. Novo, and K. Parashar. Automatic FixedPoint Conversion: a Gateway to High-Level Power Optimization. Design Automation and Test in Europe, 2014

[21] D. Ye and N. Kapre. MixFX-SCORE: Heterogeneous fixed-point compilation of dataflow computations. In Field-Programmable Custom Computing Machines, pages 206-209, 2014. 